Journal of Mathematics and Statistics 4 (4): 284-288, 2008

ISSN 1549-3644

(C) 2008 Science Publications

\title{
Estimation of the Mean of Truncated Exponential Distribution
}

\author{
Faris Muslim Al-Athari \\ Department of Mathematics, Faculty of Science, The Hashemite University, Zarqa 13115, Jordan
}

\begin{abstract}
Problem statement: In this study, the researcher considers the problem of estimation of the mean of the truncated exponential distribution. Approach: This study contracted with maximum likelihood and unique minimum variance unbiased estimators and gives a modification for the maximum likelihood estimator, asymptotic variances and asymptotic confidence intervals for the estimators. The properties of these estimators in small, moderate and large samples were investigated via asymptotic theory and computer simulation. Results: It turns out that the modified maximum likelihood estimator was more efficient than the others and exists with probability 1. Conclusion: The modified maximum likelihood estimator was always exist, fast and straightforward to compute and more likely to yield feasible values than the unique minimum variance unbiased estimator. Its variance was well approximated by the large sample variance of the other estimators.
\end{abstract}

Key words: Truncation modified maximum likelihood estimator, fisher information, simulation, exponential distribution

\section{INTRODUCTION}

Suppose that $\mathrm{X}$ be a random variable with exponential Probability Density Function (PDF) of mean $(1 / \theta)$, then the PDF of the random variable $Y$, the truncated version of $\mathrm{X}$ truncated on the right at $\mathrm{b}$, is given by:

$f(y ; \theta)=\left\{\begin{array}{lc}\theta e^{-\theta y}\left(1-e^{-\theta b}\right)^{-1}, & \text { if } 0<y \leq b \\ 0, & \text { otherwise }\end{array}\right.$

where, $b$ is a known constant.

In practice, the exponential distribution has been widely used as a model in areas ranging from studies on the lifetimes of manufactured items ${ }^{[1,2]}$ to research involving survival or remission times in chronic diseases $^{[3]}$. But in some situations, an estimate is desired of the mean among the elements of the population belonging to a certain group. For example, in life testing problems from an exponential distribution, separate estimate for the lifetime mean might be required for bulbs whose survival times are limited to be less than a constant b. In this case these survival times might follow a truncated exponential distribution. The families of truncated distributions provide densities that are useful in modeling such populations $^{[4-8]}$.

The truncated exponential distribution can occur in a variety of ways. It may directly seem to be a good fit as a distribution for a given available data set, or it may result from the type of sampling used when the underlying distribution is assumed to follow the exponential distribution ${ }^{[6,9]}$.There are different approaches for sampling selection from a subset of a larger population ${ }^{[10,11]}$.

This study deals with Maximum Likelihood estimator, (ML) and unique minimum variance unbiased estimator, (UM), of the mean of truncated exponential distribution and shows that the maximum likelihood estimator does not always exist, its existence depends upon the value of the mean of the random sample and exists with probability approaching 1 as $\mathrm{n} \rightarrow \infty$. A Modified Maximum Likelihood estimator, (MML), is considered and compared with the other estimators. The results of a large scale simulations indicate that the modified maximum likelihood estimator is more efficient and more likely to satisfy the feasibility condition, namely $0<\hat{\mu}<\mathrm{b} / 2$ for $0<\theta<\infty$.

Before proceeding with the estimation problem, it can be shown that the mean, say $\mu(\theta)$, of the truncated exponential distribution given in (1) is:

$\mu(\theta)=\frac{1}{\theta}-b\left(e^{\theta b}-1\right)^{-1}$

This function is monotonic decreasing and continuous on $(0, \infty)$ with possible range $(0, \mathrm{~b} / 2)$.

\section{MATERIALS AND METHODS}

Maximum and modified maximum likelihood estimators: Assume that $\mathrm{Y}_{1}, \mathrm{Y}_{2}, \ldots \mathrm{Y}_{\mathrm{n}}$ be a random 
sample of size $\mathrm{n}$ taken from the truncated exponential distribution given in (1). The likelihood function, say $\mathrm{L}(\theta)$ is:

$$
\mathrm{L}(\theta)=\theta^{\mathrm{n}}\left(1-\mathrm{e}^{-\theta \mathrm{b}}\right)^{-\mathrm{n}} \exp (-\mathrm{n} \theta \overline{\mathrm{y}})
$$

where, $\bar{y}$ is the sample mean. Maximizing this likelihood we get the maximum likelihood estimator for $\theta$. It follows that:

$$
\begin{aligned}
\partial \log L(\theta) / \partial \theta & =n / \theta-n b e^{-\theta b}\left(1-e^{-\theta b}\right)^{-1}-n \bar{y} \\
& =n\left[1 / \theta-b\left(e^{\theta b}-1\right)^{-1}-\bar{y}\right]
\end{aligned}
$$

Since the log-likelihood function is defined and differentiable on an open interval $(0, \infty)$, the maximum value of $L(\theta)$, if it exists, occurs at a stationary point $\theta^{*}$ at which $\partial \log \mathrm{L}(\theta) / \partial \theta=0$ and does not occur at any boundary point of the interval $(0, \infty)^{[12]}$. Setting $\partial \log \mathrm{L}(\theta) / \partial \theta=0$ us get the equation:

$1 / \theta-b\left(e^{\theta b}-1\right)^{-1}-\bar{y}=0$

It can be shown that the left-hand side of Eq. 4 is monotonic decreasing in $\theta$; as $\theta$ tends to 0 it tends to $\mathrm{b} / 2-\overline{\mathrm{y}}$ and as $\theta$ tends to infinity it tends to $(-\overline{\mathrm{y}})$.

Hence the solution to (4) is unique if it exists and it exists if and only if $0<\overline{\mathrm{y}}<\mathrm{b} / 2$ and hence when $0<\overline{\mathrm{y}}<\mathrm{b} / 2$, there exists a stationary point, say $\theta^{*}$, that satisfy Eq. 4 and $0<\theta^{*}<\infty$. Clearly $\theta^{*}$ is the unique maximum likelihood estimator of $\theta$ when $0<\bar{y}<\mathrm{b} / 2$. When $\bar{y} \geq b / 2$, the Eq. 4 does not have a solution in the domain $(0, \infty)$ and hence the likelihood function $\mathrm{L}(\theta)$ does not have a maximum. The proper definition of the ML-estimator of $\theta$ is therefore:

$\hat{\theta}=\left\{\begin{array}{l}\theta^{*}, \quad \text { if } \bar{Y}<b / 2 \\ \text { does not exist if } \bar{Y} \geq b / 2\end{array}\right.$

As $n \rightarrow \infty$, we have $\overline{\mathrm{Y}}$ converges in probability to the mean $\mu(\theta)$ of the truncated exponential p.d.f. given in (1). Because the density in (1) is monotone decreasing, a simple geometrical argument shows that the mean $\mu(\theta)$ must lie in the left half of the interval $(0$, b) and hence $\mu(\theta)<b / 2$. Then $\mathrm{P}(\overline{\mathrm{Y}}<\mathrm{b} / 2) \rightarrow 1$ as $\mathrm{n} \rightarrow \infty$, so that the MLE $\theta^{*}$ exists with probability approaching 1 as $n \rightarrow \infty$. Therefore, using the invariance property of the maximum likelihood method ${ }^{[13,14]}$, the maximum likelihood estimator, $\hat{\mu}_{1}$, of $\mu(\theta)$ is given by: $\hat{\mu}_{1}= \begin{cases}\overline{\mathrm{Y}}, & \text { if } \overline{\mathrm{Y}}<\mathrm{b} / 2 \\ \text { does not exist, } & \text { if } \overline{\mathrm{Y}} \geq \mathrm{b} / 2\end{cases}$

The same argument as before shows that the MLE estimator $\hat{\mu}_{1}$ exists with probability approaching 1 as $\mathrm{n} \rightarrow \theta$.

Under the regularity conditions ${ }^{[15-17]}$, this estimator possesses the major properties of the maximum likelihood estimator, that is $\hat{\mu}_{1}$ is consistent, asymptotic efficient and best asymptotically normal estimator with mean $\mu(\theta)$ and asymptotic variance, avar $\left(\hat{\mu}_{1}\right)$, attains the Cramer Rao lower bound.

The modification to the MLE $\hat{\mu}_{1}$, given in (5), is based on finding an estimator which is close as possible to the MLE $\hat{\mu}_{1}$ and is more likely to satisfy the feasibility condition $0<\mu(\theta)<b / 2$ than the unique minimum variance unbiased estimator. This suggested estimator, say $\hat{\mu}_{2}$, can be written as:

$\hat{\mu}_{2}= \begin{cases}\bar{Y}, & \text { if } \bar{Y}<b / 2 \\ b / 2, & \text { if } \bar{Y} \geq b / 2\end{cases}$

which corresponds to the modified maximum likelihood estimator, say $\bar{\theta}$, of $\theta$, given by:

$$
\bar{\theta}= \begin{cases}\theta^{*}, & \text { if } \bar{Y}<b / 2 \\ 0 & \text { if } \bar{Y} \geq b / 2\end{cases}
$$

The same argument as before shows that $\hat{\mu}_{1}=\hat{\mu}_{2}$ and $\hat{\theta}=\bar{\theta}$ with probability approaching 1 as $n \rightarrow \infty$.

Unique minimum variance unbiased estimator: It is obvious that the distribution in (1) represents a regular case of the exponential class of probability density functions of the continuous type and hence $\sum_{i=1}^{n} y_{i}$ is a complete sufficient statistics for $\theta$ and $\mu$. Then by using the theorem of Lehmann and Scheffe ${ }^{[13]}$, the unique minimum variance unbiased estimator, say $\hat{\mu}_{3}$, of $\mu$ is given by:

$\hat{\mu}_{3}=\bar{Y}$

The variance of $\hat{\mu}_{3}$, say $\operatorname{var}\left(\hat{\mu}_{3}\right)$, is given by:

$\operatorname{var}\left(\hat{\mu}_{3}\right)=\frac{1}{n \theta^{2}}\left[1-\zeta^{2} \mathrm{e}^{\zeta}\left(\mathrm{e}^{\zeta}-1\right)^{-2}\right]$

where, $\zeta=\theta \mathrm{b}$. 
Asymptotic variances of the estimators: The asymptotic variance of $\hat{\mu}_{1}$, say $\operatorname{avar}\left(\hat{\mu}_{1}\right)$, is the reciprocal of the Fisher information:

$$
-E\left(\partial^{2} \log L(\theta) / \partial \theta^{2}\right)\left(\frac{d \mu}{d \theta}\right)^{-2}
$$

where, $\mathrm{L}(\theta)$ is in (3) and $\mu$ is in (2). Thus:

$$
\begin{aligned}
\operatorname{avar}\left(\hat{\mu}_{1}\right) & =\frac{1}{n \theta^{2}}\left[\left[1-\zeta^{2} e^{\zeta}\left(e^{\zeta}-1\right)^{-2}\right]\right. \\
& =\operatorname{avar}(\hat{\mu} 2) \\
& =\operatorname{avar}\left(\hat{\mu}_{3}\right)
\end{aligned}
$$

Moreover, it is easy to show that $\operatorname{avar}\left(\hat{\mu}_{1}\right) \rightarrow b^{2} / 2 n$ as $\theta \rightarrow 0$.

Having obtained the asymptotic variance of $\hat{\mu}_{i}$, $\mathrm{i}=1,2,3$, the asymptotic relative efficiency, $\mathrm{ARE}_{\mathrm{ij}}$ of $\hat{\mu}_{\mathrm{i}}$ relative to $\hat{\mu}_{\mathrm{j}}$ for $\mathrm{i} \neq \mathrm{j}, \mathrm{i}=1,2,3$, is:

$$
\operatorname{ARE}_{i j}=\frac{\operatorname{avar}\left(\hat{\mu}_{\mathrm{j}}\right)}{\operatorname{avar}\left(\hat{\mu}_{\mathrm{i}}\right)}=1
$$

and the relative efficiency of $\hat{\mu}_{i}$ relative to $\hat{\mu}_{j}$ for $i \neq j$, $\mathrm{i}=1,2,3$, is defined by:

$$
\operatorname{RE}_{\mathrm{ij}}=\frac{\operatorname{MSE}\left(\hat{\mu}_{\mathrm{j}}\right)}{\operatorname{MSE}\left(\hat{\mu}_{\mathrm{i}}\right)}
$$

where, MSE is the mean-squared error.

Interval estimation of $\mu(\theta)$ : Approximate $100(1-\alpha)$ percent confidence interval for $\mu(\theta)$ in (5-7) can be constructed by the standard normal limiting distribution and the modification of Slutsky's theorem 6 given by $^{[13]}$ :

$$
\begin{aligned}
& \mathrm{P}\left\{\hat{\mu}_{\mathrm{i}}-\mathrm{z}_{\alpha / 2} \mathrm{n}^{-1 / 2}\left[\frac{1}{\theta^{* 2}}-\mathrm{b}^{2} \mathrm{e}^{\theta^{*} \mathrm{~b}}\left(\mathrm{e}^{\theta^{*} \mathrm{~b}}-1\right)^{-2}\right]^{1 / 2}<\mu(\theta)\right. \\
& \left.<\hat{\mu}_{\mathrm{i}}+\mathrm{z}_{\alpha / 2} \mathrm{n}^{-1 / 2}\left[\frac{1}{\theta^{* 2}}-\mathrm{b}^{2} \mathrm{e}^{\theta^{*} \mathrm{~b}}\left(\mathrm{e}^{\theta^{*} \mathrm{~b}}-1\right)^{-2}\right]^{1 / 2}\right\} \\
& =1-\alpha, \quad \text { when } \overline{\mathrm{Y}}<\mathrm{b} / 2
\end{aligned}
$$

and

$$
\begin{aligned}
& \mathrm{P}\left(\hat{\mu}_{\mathrm{i}}-\mathrm{z}_{\alpha / 2} \frac{\mathrm{b}}{\sqrt{2 \mathrm{n}}}<\mu(\theta)<\hat{\mu}_{\mathrm{i}}-\mathrm{z}_{\alpha / 2} \frac{\mathrm{b}}{\sqrt{2 \mathrm{n}}}\right)=1-\alpha, \\
& \text { when } \overline{\mathrm{Y}} \geq \mathrm{b} / 2
\end{aligned}
$$

for all $\mathrm{i}=1,2,3$, where $\mathrm{z}_{\alpha / 2}$ is the $100(1-\alpha)$ percent point of the standard normal distribution.

The simulation technique: In order to investigate the properties and the values of the estimators $\hat{\mu}_{1}, \hat{\mu}_{2}$ and $\hat{\mu}_{3}$ a large scale simulation investigation was made for the exponential p.d.f. truncated on the right. To get the biases, variances and the mean-squared errors numerically, the simulation technique with the help of MATLAB, the language of technical computing version 6.5 is used $^{[18]}$. These are computed for 50,000 samples of sizes $(n=20,30,50,100,200)$ generated from the truncated exponential distribution. Pseudo-random uniform numbers were obtained from the function RAND of the MATLAB. The transformation to the truncated exponential distributed variable is given by:

$$
\begin{aligned}
\mathrm{Y}_{\mathrm{i}} & =\mathrm{F}^{-1}\left(\mathrm{U}_{\mathrm{i}}\right) \\
& =-\frac{1}{\theta} \log \left[1-\mathrm{U}_{\mathrm{i}}\left(1-\mathrm{e}^{-\zeta}\right)\right]
\end{aligned}
$$

Where:

$F()=$. The distribution function of the truncated exponential random variable

$\mathrm{Ui}=$ Uniformly distributed random variable on $(0,1)^{[10]}$

For each combination of $(n, \zeta), 50,000$ trials have been done to find 50,000 values of each estimator. These estimators are then used to estimate the means, the values of the biases, the variances and the meansquared errors for each estimator. A computer simulation experiment was run to compare three methods of estimation of the mean of truncated exponential distribution. Simulations were performed for sample sizes $\mathrm{n}=20,30,50,100,200$ with the truncation points taking values $\zeta=0.05,0.25,0.5$, 1.0(1.5)10.0. For each combination of values of $n$ and $\zeta, 50,000$ random samples were generated from the truncated exponential distribution and for each sample the mean $\mu(\theta)$ was estimated by each of the three methods: (a) the method of Maximum Likelihood (ML), described before; (b) the method of Modified Maximum Likelihood (MML); and (c) the method of Unique Minimum variance unbiased estimator (UM).

\section{RESULTS}

The simulation results for estimation of the mean of truncated exponential distribution are shown in Table 1-3. 
J. Math. \& Stat., 4 (4): 284-288, 2008

Table 1: Percentage of the absolute values of the biases of the estimators ML and MML for $\theta=1$

\begin{tabular}{|c|c|c|c|c|c|c|c|c|c|c|c|}
\hline \multicolumn{12}{|c|}{$\zeta$} \\
\hline $\mathrm{n}$ & Method & 0.05 & 0.25 & 0.5 & 1.0 & 2.5 & 4.00 & 5.50 & 7.00 & 8.50 & 10.0 \\
\hline \multirow[t]{2}{*}{20} & ML & 0.24 & 0.97 & 1.42 & 1.24 & 0.01 & 0.04 & 0.04 & 0.03 & 0.03 & $\overline{0.02}$ \\
\hline & MML & 0.12 & 0.42 & 0.51 & 0.29 & 0.03 & 0.04 & 0.04 & 0.03 & 0.03 & 0.02 \\
\hline \multirow[t]{2}{*}{30} & ML & 0.20 & 0.75 & 0.98 & 0.62 & 0.07 & 0.09 & 0.09 & 0.08 & 0.08 & 0.08 \\
\hline & MML & 0.09 & 0.30 & 0.31 & 0.10 & 0.07 & 0.09 & 0.09 & 0.08 & 0.08 & 0.08 \\
\hline \multirow[t]{2}{*}{50} & ML & 0.15 & 0.51 & 0.56 & 0.18 & 0.07 & 0.09 & 0.10 & 0.10 & 0.08 & 0.10 \\
\hline & MML & 0.07 & 0.19 & 0.15 & 0.00 & 0.07 & 0.09 & 0.10 & 0.10 & 0.10 & 0.10 \\
\hline \multirow[t]{2}{*}{100} & ML & 0.10 & 0.30 & 0.20 & 0.01 & 0.01 & 0.00 & 0.01 & 0.01 & 0.02 & 0.02 \\
\hline & MML & 0.05 & 0.10 & 0.05 & 0.00 & 0.01 & 0.00 & 0.01 & 0.01 & 0.02 & 0.02 \\
\hline \multirow[t]{2}{*}{200} & ML & 0.07 & 0.14 & 0.05 & 0.01 & 0.01 & 0.02 & 0.03 & 0.03 & 0.03 & 0.03 \\
\hline & MML & 0.03 & 0.04 & 0.01 & 0.01 & 0.01 & 0.02 & 0.03 & 0.03 & 0.03 & 0.03 \\
\hline
\end{tabular}

Table 2: Percentage values of the (n $\left.\theta^{2}\right)$ var of the ML, MML and UM estimators and the (n $\left.\theta^{2}\right)$ avarun of ML and UM estimators for $\theta=1$

\begin{tabular}{|c|c|c|c|c|c|c|c|c|c|c|c|}
\hline & & & & & & & & & & & \\
\hline $\mathrm{n}$ & Method & 0.05 & 0.25 & 0.5 & 1.0 & 2.5 & 4.0 & 5.5 & 7.0 & 8.5 & 10.0 \\
\hline 20 & ML & 0.01 & 0.23 & 1.1 & 5.6 & 39.0 & 69.7 & 87.6 & 95.5 & 98.4 & 99.4 \\
\hline & MML & 0.01 & 0.25 & 1.3 & 6.6 & 39.2 & 69.7 & 87.6 & 95.5 & 98.4 & 99.4 \\
\hline & UM & 0.02 & 0.52 & 2.1 & 7.9 & 39.1 & 69.6 & 87.5 & 95.5 & 98.5 & 99.5 \\
\hline 30 & ML & 0.01 & 0.24 & 1.2 & 6.2 & 39.3 & 70.0 & 87.8 & 95.7 & 98.6 & 99.6 \\
\hline & MML & 0.01 & 0.26 & 1.4 & 6.2 & 39.4 & 70.0 & 87.8 & 95.7 & 98.6 & 99.6 \\
\hline & UM & 0.02 & 0.52 & 2.1 & 7.9 & 39.1 & 69.6 & 87.5 & 95.5 & 98.5 & 99.5 \\
\hline 50 & ML & 0.01 & 0.25 & 1.3 & 7.0 & 39.1 & 69.6 & 87.5 & 95.5 & 98.5 & 99.5 \\
\hline & MML & 0.01 & 0.29 & 1.6 & 7.6 & 39.1 & 69.6 & 87.5 & 95.5 & 98.5 & 99.5 \\
\hline & UM & 0.02 & 0.52 & 2.1 & 7.9 & 39.1 & 69.6 & 87.5 & 95.5 & 98.5 & 99.5 \\
\hline 100 & ML & 0.01 & 0.28 & 1.5 & 7.7 & 39.0 & 69.4 & 87.2 & 95.1 & 98.1 & 99.1 \\
\hline & MML & 0.01 & 0.33 & 1.8 & 7.9 & 39.0 & 69.4 & 87.2 & 95.1 & 98.1 & 99.1 \\
\hline & UM & 0.02 & 0.52 & 2.1 & 7.9 & 39.1 & 69.6 & 87.5 & 95.5 & 98.5 & 99.5 \\
\hline 200 & ML & 0.01 & 0.33 & 1.8 & 7.9 & 39.1 & 69.6 & 87.5 & 95.5 & 98.5 & 99.5 \\
\hline & MML & 0.01 & 0.39 & 2.0 & 7.9 & 39.1 & 69.6 & 87.5 & 95.5 & 98.5 & 99.5 \\
\hline & UM & 0.02 & 0.52 & 2.1 & 7.9 & 39.1 & 69.6 & 87.5 & 95.5 & 98.5 & 99.5 \\
\hline & avar & 0.02 & 0.52 & 2.1 & 7.9 & 39.1 & 69.6 & 87.5 & 95.5 & 98.5 & 99.5 \\
\hline
\end{tabular}

Table 3: Percentage values of the relative efficiencies of the ML and UM estimators relative to the MML estimator

\begin{tabular}{|c|c|c|c|c|c|c|c|c|c|c|c|}
\hline & & & & & $\zeta$ & & & & & & \\
\hline $\mathrm{n}$ & Method & 0.05 & 0.25 & 0.5 & 1.0 & 2.5 & 4.0 & 5.5 & 7.0 & 8.5 & 10.0 \\
\hline \multirow[t]{2}{*}{20} & ML & 16.9 & 67.70 & 89.2 & 112.8 & 100.6 & 100.0 & 100.0 & 100.0 & 100.0 & 100.0 \\
\hline & UM & 50.3 & 54.20 & 63.6 & 83.8 & 100.2 & 100.2 & 100.0 & 99.9 & 99.9 & 99.9 \\
\hline \multirow[t]{2}{*}{30} & ML & 53.1 & 72.00 & 97.3 & 112.7 & 100.1 & 100.0 & 100.0 & 100.0 & 100.0 & 100.0 \\
\hline & UM & 50.1 & 55.80 & 68.4 & 90.4 & 100.6 & 100.5 & 100.3 & 100.0 & 100.1 & 100.1 \\
\hline \multirow[t]{2}{*}{50} & ML & 54.4 & 80.20 & 107.2 & 107.8 & 100.0 & 100.0 & 100.0 & 100.0 & 100.0 & 100.0 \\
\hline & UM & 49.9 & 58.90 & 75.9 & 95.6 & 99.9 & 100.0 & 100.0 & 100.0 & 99.9 & 99.9 \\
\hline \multirow[t]{2}{*}{100} & ML & 56.8 & 94.00 & 112.8 & 101.6 & 100.0 & 100.0 & 100.0 & 100.0 & 100.0 & 100.0 \\
\hline & UM & 50.5 & 66.10 & 87.3 & 99.2 & 99.7 & 99.7 & 99.6 & 99.6 & 99.6 & 99.6 \\
\hline \multirow[t]{2}{*}{200} & ML & 60.2 & 107.30 & 107.3 & 100.1 & 100.0 & 100.0 & 100.0 & 100.0 & 100.0 & 100.0 \\
\hline & UM & 52.0 & 76.30 & 96.1 & 99.8 & 100.0 & 100.0 & 100.0 & 100.0 & 100.0 & 100.0 \\
\hline
\end{tabular}

\section{DISCUSSION}

Apart from the case $\zeta>2.5$ when all the estimation methods have comparable performance, Table 1 shows that the MML estimator has, consistently, the lowest absolute bias of the two biased estimators of $\mu$, its advantage being particularly marked in small samples $\mathrm{n}=20,30$ and in moderate samples $\mathrm{n}=50$. Table 2 shows that the MML estimator has slightly larger variance than the ML estimator when $\zeta<2.5$, but its variance is small and in most cases relatively insignificant compared to the bias in its contribution to the mean-squared error. The UM estimator has the largest variance of the three estimators of $\mu$ when $\zeta<2.5$. The variance of the MML estimator is well approximated by the asymptotic variance of ML and UM estimators given by (9) and the last line of Table 2. Table 3 gives the percentage values of the relative efficiencies of ML and UM estimators defined as the ratio of the means square errors, relative to the 
MML estimator. It is obvious from this table that, in general, the ML and the UM estimators are less efficient than the MML especially when $\zeta<2.5$ and their relative efficiencies increase with $\zeta$.

\section{CONCLUSION}

Estimations of the mean of truncated exponential distribution have been suggested and their properties are studied. It turns out that the modified maximum likelihood estimator has several advantages over the other estimators. It is always exist, fast and straightforward to compute and more likely to yield feasible values for the estimated mean than the unique minimum variance unbiased estimator. The bias of the estimator is small and decreases rapidly as the sample size increases. The variance of the MML estimator is comparable with those of the ML and UM estimators. The variance of the MML estimator is well approximated by the large sample variance of ML and UM estimators.

\section{REFERENCES}

1. Davis, D.J., 1952. An analysis of some failure data. J. Am. Stat. Assoc., 47: 113-150. http://www.jstor.org/pss/2280740

2. Epstein, B., 1958. The exponential distribution and its role in life-testing. Ind. Q. Control, 15: 2-7. http://oai.dtic.mil/oai/oai?verb=getRecord\&metada taPrefix $=$ html\&identifier $=$ AD0158605

3. Feigl, P. and M. Zelen, 1965. Estimation of exponential survival probabilities with concomitant information. Biometric, 21: 826-838. http://www.jstor.org/pss/2528247

4. Ahmad, A.A., 2001. Moments of order Statistics from doubly truncated continuous distributions. Statistics, 35: 479-494. DOI: 10.1080/02331880108802749

5. Ahmad, A.A. and M. Fawzy, 2003. Recurrence relations for single moments of generalized order statistics from doubly truncated distributions. J. Stat. Plann. Inference, 117: 241-249. DOI: $10.1016 / \mathrm{s} 0378-3758(02) 00385-3$
6. Bain, L.J. and G. Gaoxiong, 1996. Conditional maxima and inferences for the truncated exponential distribution. Can. J. Stat., 24: 251-256. http://www.jstor.org/pss/3315630

7. Joshi, P.C., 1979. A note on the moments of order Statistics from doubly truncated exponential distribution. Ann. Inst. Stat. Math., 31: 321-324. DOI: $10.1007 / \mathrm{BF} 02480290$

8. Khan, A.H. and M.M. Ali, 1987. Characterization of probability distributions through higher order gap. J. Commun. Stat. Theor. Math., 16: 1281-1287. DOI: $10.1080 / 03610928708829438$

9. Deemer, W.L. and F.V. David, 1955. Estimation of parameters of truncated or censored exponential distributions. Anal. Math. Stat., 26: 498-504. DOI: 10.1214/aoms/1177728494

10. Tryfos, P., 1996. Sampling Methods for Applied Research. John Wiley and Sons, New York, ISBN: 978-0-471-04727-8, pp: 464.

11. Wooldridge, J.M., 2002. Econometric Analysis of Cross Section and Panel Data. 2nd Edn., MIT Press, Cambridge, London, ISBN: 13: 978-0-26223219-7, pp: 784.

12. Anton, H., I. Bivens and S. Davis, 2005. Calculus Single Variable. 8th Edn., John Wiley and Sons, New York, ISBN: 0-47148273-0.

13. Hogg, R.V. and T.C. Allen, 1995. Introduction to Mathematical Statistics. 5th. Edn., Prentice-Hall Inc., New Jersey, ISBN: 10:0023557222, pp: 564.

14. Johnson, R.A. and W.W. Dean, 1998. Applied Multivariate Statistical Analysis. 4th Edn., Prentice-Hall, Inc., New Jersey, ISBN: 0-13834194-x, pp: 799.

15. Johnson, J. and J. Dinardo, 1997. Econometric Methods. McGraw-Hill, New York, ISBN: 0070327203, pp: 480.

16. Verbeek Marno, 2000. A Guide to Modern Econometrics. John Wiley and Sons, New York, ISBN: 978-0-470-51769-7.

17. Zacks, S., 1971. The Theory of Statistical Inference. John Wiley and Sons, New York, ISBN: 0471981036, pp: 626.

18. Enander, E.P., A. Sjoberg, B. Melin and P. Isaksson, 1996. The MATLAB, Hand Book. Addison Wesley, Longman, ISBN: 0-201-87757-0. 